\title{
Mathematical Optimization and Algorithms for Offshore Wind Farm Design: An Overview
}

\author{
Martina Fischetti - David Pisinger
}

Received: 14 November 2017 / Accepted: 20 February 2018/Published online: 30 April 2018

(C) Springer Fachmedien Wiesbaden GmbH, part of Springer Nature 2018

\begin{abstract}
Wind energy is a fast evolving field that has attracted a lot of attention and investments in the last decades. Being an increasingly competitive market, it is very important to minimize establishment costs and increase production profits already at the design phase of new wind parks. This paper is based on many years of collaboration with Vattenfall, a leading wind energy developer and wind power operator, and aims at giving an overview of the experience of using Mathematical Optimization in the field. The paper illustrates some of the practical needs defined by energy companies, showing how optimization can help the designers to increase production and reduce costs in the design of offshore parks. In particular, the study gives an overview of the individual phases of designing an offshore wind farm, and some of the optimization problems involved. Finally it goes in depth with three of the most important optimization tasks: turbine location, electrical cable routing and foundation optimization. The paper is concluded with a discussion of future challenges.
\end{abstract}

Keywords Offshore wind farm design - Mathematical optimization - Mixed integer linear programming .

Heuristics · Cable routing · Wind farm layout · Jacket structure optimization

Accepted after one revision by Prof. Dr. Suhl.

M. Fischetti

Vattenfall BA Wind, Jupitervej 6, 6000 Kolding, Denmark

e-mail: martina.fischetti@vattenfall.com

D. Pisinger $(\bowtie)$

DTU Management Engineering, Technical University of Denmark, Produktionstorvet 424, 2800 Kongens Lyngby, Denmark

e-mail: dapi@dtu.dk

\section{Introduction}

Environmental sustainability asks for a considerable reduction in the use of fossil fuels, looking to alternative sources of energy. As a consequence, increasingly more energy companies are investing, for example, in wind energy, creating a more competitive market for renewable energy. Particular attention is given to offshore solutions (wind parks located at sea). In this paper we will give a detailed overview of how the offshore wind park design is carried out in wind-energy companies, focusing on how mathematical optimization techniques can make an impact in reducing costs and increase production. We will mainly address the optimization tasks related to the design phase of a wind park. This is the initial phase in defining a new wind park, so there is more room for optimization. In particular, we focus on three specific problems - wind turbines location, connection of offshore turbines with cables and turbine foundation design - as these are some of the optimization tasks having the greatest impact. Our models make it possible to systematically organize and process input data from different sources, as well as intuitively communicate the output to the decision makers. Therefore, they can be easily linked to Vattenfalls information system, both to gather data and to store optimized solutions and other strategic analysis built on top of them.

\subsection{Wind Park Design Phases}

In this overview paper we will focus on the design phase of offshore wind parks. Designing a wind park is a complex project, involving different expertises and a large number of optimization tasks. Most of the main optimization tasks of the problem are still not totally automated and commercial software ignores several important constraints. 
Generally speaking, the main steps in the design of a wind park consist of:

- site selection: often decided by the government and put on tender;

- data collection: most of it is performed previous to the tender;

- technology selection: which includes, for example, selecting the manufacturer and the model for all the components of a wind park (e.g., selecting which turbines to consider for the park);

- definition of the layout: deciding where to locate the turbines in the site;

- evaluation of foundation costs and soil conditions;

- cable routing: deciding how to connect the turbines to the substation(s);

- electrical studies: defining the detailed electrical design, dimensioning equipment, computing power losses, proving compliance to grid codes, voltage levels and frequency limits in the connection to the grid;

- design of each specific foundation (for each selected location).

According to our experience, the design of a wind park is structured as follows. When a company decides to enter a tender to construct a new wind park in Europe, it generally receives an area (selected by the government) and GIS information about it, e.g., the wind statistics measured on the site, the seabed conditions, possible obstacles in the site, etc. The company can decide what turbine type to build in the site and where to locate the turbines within the boundaries of the given area. The total Megawatt (MW) production of the site is also given at tender phase, as the grid operator needs to ensure stability when the new park production is injected in the existing power grid system. Since only one type of turbine is built in each site (mainly for maintenance reasons), this MW restriction easily translates into a fixed number of turbines that can be built in the site. With all this information at hand, the first task that the company engineers normally face is to decide where to locate the turbines (i.e., the wind farm layout optimization problem). This is a very challenging task due to the so-called wake effect. The wake effect is the interference phenomenon for which, if two turbines are located one close to another, the upwind one creates a shadow on the downstream turbine (see Fig. 1). This is of great importance in the design of the layout since it results in a loss of power production for the turbine downstream, which is also subject to a possibly strong turbulence. It is estimated in Barthelmie et al. (2009) that, in large offshore wind farms, the average power loss due to turbine wakes is around $10-20 \%$ of the total energy production. It is then obvious that power production can increase significantly if the park layout is designed so as to reduce the wake effect

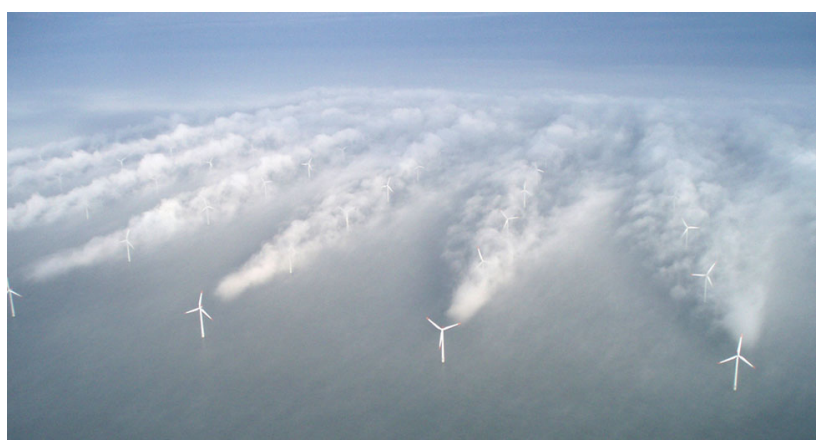

Fig. 1 Wake effect in an offshore wind park (Vattenfall)

as much as possible. As we will see, mathematical optimization can successfully be used at this stage.

Once the turbine positions are decided, the layout is generally forwarded to the electrical team. Offshore turbines need to be connected to shore with cables. The turbines are connected with lower voltage cables to an offshore substation where all the energy is collected - this is the so called inter-array cable connection. A unique high-voltage cable (called export cable) is used to transport the energy from the substation to shore. The substation and export cable can be established by the same company that constructs the park or can be established before tendering. In this paper we assume the second scenario, so substation(s) and export cable are assumed to be fixed a-priori. The offshore inter-array cable routing problem consists of finding the minimum cost connection of all offshore turbines. Different types of cables, with different capacities, electrical resistances and prices, can be used. This optimization task is still carried out manually in many companies, leading to highly suboptimal cable routes. As we will see, Mixed Integer Linear Programming (MILP) and ad-hoc heuristics can be used to solve the inter-array cable routing problem. Considering cable losses when designing the cable route is also very important. Due to the resistance in the cables, indeed, some energy gets lost in the transmission to the substation. An optimized selection of the cable structure and the cable type, can reduce the amount of current losses over the lifetime of the park.

While the electrical team works on the cable routing, another team works on the turbine foundations. Once the turbine position is identified, the specific locations are checked for sea bed conditions. Depending on the environmental conditions at each position, the water depth and the turbine type selected, different foundations can be designed for each turbine. Currently, the most used foundation type is the monopile, which is the simplest foundation available on the market. When the water is very deep, more complex structures need to be used as, for example, jacket foundations. Different optimization tasks can be identified in the foundation design, especially 
looking at the component selection. Here, the main challenge is to ensure that the foundation will be able to stand the different forces acting on it, due to the turbine movements but also the sea conditions (waves, currents and so on).

\subsection{Literature Overview}

In this subsection we give a literature study of different optimization problems that may arise in the establishment and operation of an offshore wind park. In the following sections we will then go in depth with some of the most important optimization tasks when establishing an offshore wind farm.

Probably one of the most studied optimization tasks in the wind park design is the wind farm layout problem. As we will see in further details later, this is a very challenging task due to the wake effect.

The wind farm layout problem was first formulated as an optimization model in the master thesis (Fagerfjall 2010). The objective is to position wind turbines taking into account wake effect and sound limitations for surrounding areas. The work of Turner et al. (2014) also develops a mathematical programming framework for the wind farm layout problem, focusing on the wake effect modelling. The resulting nonlinear optimization model is approximated both as a quadratic integer program and as a mixedinteger linear program. Only a limited number of wind scenarios are considered in the paper. The paper Zhang et al. (2014) focuses on better capturing the nonlinearities of the wake effect, proposing a constraint programming and a mixed integer programming version of the model. Decomposition techniques are used to improve solution complexity. A continuous approach to the wind farm layout problem has been used in Kwong et al. (2012) and Kusiak and Song (2010). The continuous models are highly nonconvex and turn out to be intractable from a computational viewpoint when considering real-world cases, especially when considering obstacles in the site.

The models presented in this overview have their origin in the MILP formulation of Archer et al. (2011). In Fischetti and Monaci (2016) the formulation was extended, paving the way for an easier stochastic version (taking different wind scenarios into account). This MILP model, with some ad-hoc heuristics, is able to solve large instances.

The next problem in the design phase of a wind park is the cable routing of offshore parks. This task consists in finding the optimal connection among offshore turbines and some collection points at sea, i.e., the so-called substations. Bauer and Lysgaard (2015) proposed a model based on an open vehicle routing problem formulation. The model assumes that only one cable can enter a turbine, a condition that is seldom met in real-world cases. Different solution approaches were proposed in Berzan et al. (2011), where a divide-and-conquer heuristic and an integer programming model were presented and tested on small instances. Furthermore, Dutta and Overbye (2011) presented a clustering heuristic for cable routing. Finally, matheuristic approaches have proven to be very valuable in real-world applications, especially when taking losses into account Fischetti and Pisinger (2017c).

Another important set of problems in offshore wind farm optimization regards the maintenance of offshore parks. An offshore wind farm demands frequent maintenance to avoid breakdown and production losses. Maintenance requires expensive resources, such as vessels or helicopters, so it is important to use them effectively. Optimization of vessel routing and of maintenance scheduling was studied in Dai et al. (2015), while Gundegjerde et al. (2015) was focused on the optimization of the fleet size, proposing a stochastic three-stage programming model. Gutierrez-Alcoba et al. (2017) used bi-level optimization to cope with real-time requests. On the first (tactical) level, the fleet composition for a certain time horizon is decided, while on the second (operational) level, its operations schedule is optimized, given failures and actual weather conditions. Decomposition methods were instead used by Irawan et al. (2017) to find the optimal schedule for maintaining the turbines, the optimal routes for the crew transfer vessels, and the number of technicians required for each vessel. The routes take several constraints into account such as weather conditions, the availability of vessels, and the number of technicians available at the base.

Other optimization challenges concern the structure of the turbine itself. Wind turbines are, indeed, very expensive engineering systems subject to high loads. Turbine towers, support structures and foundation systems can be optimized in order to reduce costs while ensuring no damages in the overall structure. Muskulus and Schafhirt (2014) gave an overview of the topic and of the literature in the field. Oest et al. (2017) focused on the optimization of a specific foundation type, i.e., jacket foundations. Jacket foundations are one of the most complex/expensive structures, normally used at high water depth or at difficult soil conditions.

Finally, optimization of energy storage is getting still more attention in the wind energy sector. Due to variation in production and electricity prices, it can be beneficial to store the produced energy in order to sell it when production is lower and prices are higher. This helps stabilizing the grid, but can also increase the profit of wind farms. In Hou et al. (2017) it was investigated how to couple an offshore wind farm with hydrogen storage. The resulting non-linear optimization model was solved using 
sequential quadratic programming methods and particle swarm optimization. Another solution to the variability of wind power, is to use hybrid systems, i.e., to compensate the wind energy downtimes with other energy sources. One example, is to use solar energy: Sinha and Chandel (2015) gave an overview of optimization methods for the integration of photovoltaic and wind energy: mostly hybrid techniques and metaheuristics have been used for this task.

As offshore wind farms are getting older, we will in the coming years see an increased need for decommissioning the farms. Not much work has been done on optimizing this phase. Topham and McMillan (2017) gave an overview of the tasks involved. These tasks include removing wind turbines, foundations, substations and cables, as well as onshore installations. Interesting optimization problems to be considered in decommissioning could be planning of the individual phases as well as transportation planning. Hou et al. (2016) presented an optimization model for the decommissioning, in which the foundations are reused, but turbines are replaced with newer models. The problem was solved through particle swarm optimization.

\subsection{Outline of the Paper}

In the next sections we go in depth with three of the most important optimization tasks being part of designing an offshore wind farm. These tasks are the turbine location, the electrical cable routing, and finally optimization of foundations. The problem formulations and solution methods are based on our experience in collaborating with a leading energy company in wind farm design. Section 2 is dedicated to wind farm layout optimization and illustrates how to use mathematical optimization techniques to solve this challenging optimization task. Section 3 focuses on offshore wind farm cable routing optimization. A mathematical formulation of the problem is presented and matheuristics approaches are developed for solving the model. Several real-world examples are considered in Sect. 3.1. Finally in Sect. 3.2 we show how power losses can be handled in the optimization. Section 4 is dedicated to the optimization of jacket foundations and is an original contribution of this paper. In particular, Sect. 4.1 shows how to model the optimization task using MILP models, while Sect. 4.2 illustrates the potential of this optimization on a case study. Finally, Sect. 5 concludes the overview and proposes directions for future research. Sections 2 and 3 are based on Fischetti and Monaci (2016) and Fischetti and Pisinger (2017c), with some extensions (e.g., considering cost of foundations in the layout optimization) and several additional real-world examples.

\section{A Proximity Search Heuristic for Wind Farm Layout}

In this section we will describe solution methods for the offshore wind farm layout problem. This problem consists in finding an optimal allocation of wind turbines in order to maximize power production, taking the wake effect into account. The building area (site) and its resource maps are given on input. The optimizer considers:

(a) a minimum and maximum number of turbines that can be built;

(b) a minimum separation distance between any pair of turbines to ensure that the blades do not physically clash;

(c) the interference between installed turbines (wake effect).

This problem is very challenging due to the large number of possible positions, which can exceed 20,000 in realworld applications. Fischetti and Monaci (2014) and Fischetti (2014) underline the importance of having a suitable formulation of the MILP model and MILP-based heuristics on top of it, for such a large-size problem. In the following we will briefly summarize this work.

The available sea area to construct the wind farm can be discretized in a number of possible positions by over-imposing a regular grid. Let $V$ denote the set of all possible positions for a turbine and let

- $I_{i j}$ be the interference (loss of power) experienced by site $j$ when a turbine is installed at site $i$, with $I_{j j}=0$ for all $j \in V$; Jensen's model Jensen (1983) can be used to compute the interference;

- $P_{i}$ be the power that a turbine would produce if built alone at position $i$;

- $N_{\min }$ and $N_{\max }$ be the minimum and maximum number of turbines that can be built, respectively;

- $D_{\min }$ be the minimum distance between two turbines;

- $\operatorname{dist}(i, j)$ be the distance between sites $i$ and $j$.

In addition, let $G_{I}=\left(V, E_{I}\right)$ denote the incompatibility graph with

$E_{I}=\left\{(i, j): i, j \in V, \operatorname{dist}(i, j)<D_{\min }, i \neq j\right\}$

and let $n=|V|$ denote the total number of positions.

In Fischetti and Monaci (2016), a binary variable $x_{i}$ is defined for each $i \in V$ to be 1 if and only if a turbine is built at position $i \in V$. The original quadratic objective function (to be maximized)

$\sum_{i \in V} P_{i} x_{i}-\sum_{i \in V}\left(\sum_{j \in V} I_{i j} x_{j}\right) x_{i}$

is restated as 
$\sum_{i \in V}\left(P_{i} x_{i}-w_{i}\right)$

where the variable $w_{i}$ is defined as

$w_{i}=\left(\sum_{j \in V} I_{i j} x_{j}\right) x_{i}= \begin{cases}\sum_{j \in V} I_{i j} x_{j} & \text { if } x_{i}=1 \\ 0 & \text { if } x_{i}=0\end{cases}$

and denotes the total interference caused by site $i$. The model then reads

$\max \quad z=\sum_{i \in V}\left(P_{i} x_{i}-w_{i}\right)$

s.t. $\quad N_{\min } \leq \sum_{i \in V} x_{i} \leq N_{\max }$

$x_{i}+x_{j} \leq 1 \quad(i, j) \in E_{I}$

$\sum_{j \in V} I_{i j} x_{j} \leq w_{i}+M_{i}\left(1-x_{i}\right) \quad i \in V$

$x_{i} \in\{0,1\} \quad i \in V$

$w_{i} \geq 0 \quad i \in V$

The objective function (3) maximizes the total power production by taking interference losses into account. Constraints (4) impose a minimum and a maximum number of turbines that can be constructed in the site, while (5) ensure the minimum distance between turbines. Constraints (6) relate variables $w_{i}$ with interference. A big-M term $M_{i}$ is used to deactivate the constraint in case $x_{i}=0$, namely

$M_{i}=\sum_{j \in V,(i, j) \notin E_{I}} I_{i j}$.

Finally (7) and (8) define our binary and continuous variables, respectively.

As shown in details in Fischetti and Monaci (2016), using a single index variable $w_{i}$ allows this model to solve larger instances compared with equivalent two-index models in the literature (e.g., Archer et al. 2011; Fagerfjall 2010). Another strength of this formulation is the ability of easily dealing with different wind scenarios. Indeed, the definition of the turbine power $P_{i}$ and of the interference $I_{i j}$ depends on the wind scenario considered, which greatly varies in time. Using statistical data, one can collect a large number, say $K$, of wind scenarios $k$, each associated with $P_{i}^{k}, I_{i, j}^{k}$ and with arising probability $\pi_{k}$. Using that data, one can write a stochastic programming variant of the previous model where only the objective function needs to be modified as

$$
z=\sum_{k=1}^{K} \pi_{k}\left(\sum_{i \in V} P_{i}^{k} x_{i}-\sum_{i \in V} \sum_{j \in V} I_{i j}^{k} x_{i} x_{j}\right)
$$

while all constraints stay unchanged as they only involve "first-stage" variables $x$. It is therefore sufficient to define
$P_{i}:=\sum_{k=1}^{K} \pi_{k} P_{i}^{k} \quad i \in V$

$I_{i j}:=\sum_{k=1}^{K} \pi_{k} I_{i j}^{k} \quad i, j \in V$

to obtain the same model (1)-(8) as before. Therefore, using this MILP formulation together with Jensen's model for wake effect, one can easily address the realistic situation in which many wind scenarios are considered, just by using a suitable definition of the input data; this is not the case for more sophisticated wake effect models, typically leading to really huge stochastic programming variants.

As shown in details in Fischetti (2014), this model is not suitable for large real-world instances, and a heuristic framework must be built around it.

The authors showed that large-scale instances (around 20,000 possible positions to locate turbines) can be solved on a standard PC, using some ad-hoc heuristics and a MILP-based heuristic scheme called Proximity Search (Fischetti and Monaci 2016).

When facing large-size problem it is standard practice to "warm start" the MILP solver, using a first heuristic solution (let us call it $(\tilde{x}, \tilde{w})$ ) to initialize the incumbent of the solver. However, it is often seen in practice [see e.g., Boschetti et al. (2009)] that this strategy is unlikely to produce improved solutions within acceptable computing times if the underlying MILP model is very large and the formulation is weak - as it happens in our context. So, a different use of the MILP solver is suggested, which is used to "search a neighborhood" of the heuristic solution $(\tilde{x}, \tilde{w})$, as in the so-called "Proximity Search" method (Fischetti and Monaci 2014). In the wind farm context, some simple ad-hoc heuristics are used to generate a first solution and then the MILP solver is used as a black-box to improve this first solution $(\tilde{x}, \tilde{w})$ in stages. At each stage, an explicit cutoff constraint

$\sum_{i \in V}\left(P_{i} x_{i}-w_{i}\right) \geq \sum_{i \in V}\left(P_{i} \tilde{x}_{i}-\tilde{w}_{i}\right)+\theta$

is added to the original MIP, where $\theta>0$ is a given tolerance that specifies the minimum improvement required. The objective function of the problem can then be replaced by a new "proximity function" (to be minimized):

$$
\Delta(x, \tilde{x})=\sum_{j \in V: \tilde{x}_{j}=0} x_{j}+\sum_{j \in V: \tilde{x}_{j}=1}\left(1-x_{j}\right)
$$

This function measures the Hamming distance between a generic binary vector $x$ and the given $\tilde{x}$ (note that continuous variables $w_{i}$ 's play no role in this definition). One then applies the MILP solver, as a black box, to the modified problem in the hope of finding an improved solution having a small Hamming distance from $\tilde{x}$. The computational 
experiments in Fischetti and Monaci (2014) confirm that this approach is quite successful. The proximity objective function is indeed beneficial both in speeding up the solution of the LP relaxations, and in driving the heuristics embedded in the MILP solvers. This method proved to be particularly valuable for the wind farm layout problem.

\subsection{Real-World Application}

Using mathematical optimization techniques to optimize the turbine location can lead to huge savings. We used the optimization framework outlined in the previous section on a real wind park in The Netherlands. The Borssele area, in the Dutch province of Zeeland, was selected to construct a new wind park in 2016. The big offshore area was divided in four sites (Fig. 2), and put on tender in two stages. In the first stage (summer 2016), sites I and II were on tender, for a combined 700-760 MW capacity. Here we will consider one of the two, namely Borssele I (350 MW capacity).

The borders of the area were given at tender phase, as shown in Fig. 2. Part of the area was actually not available to construct turbines, due to pre-existing cables in the seabed: Fig. 3 shows the area available to place turbines. It can be noticed that two corridors are forbidden. These kinds of "obstacles" are quite common in real sites, but they are easy to handle by our discrete model (by simply removing forbidden positions from the least of possible positions on input). In our experiment we were asked to locate $507 \mathrm{MW}$ turbines (154 m rotor diameter) in the area. The company specified a minimum distance between

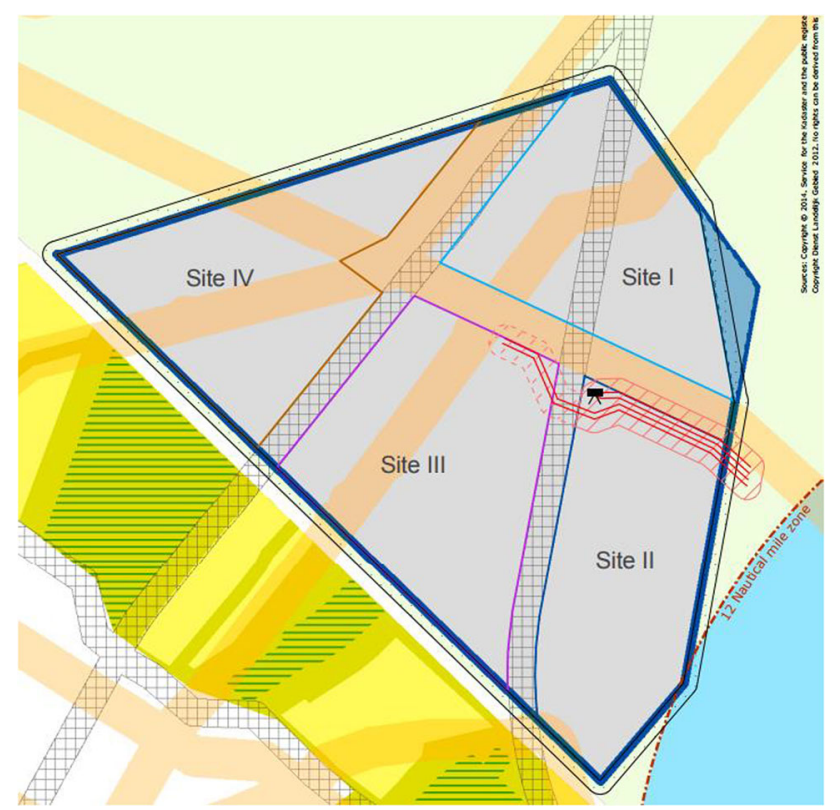

turbines of five rotor diameters. $60,000+$ wind scenarios were defined from real wind measurements in the site. The outcome of our optimization model is shown in Fig. 3: the red dots represent built turbines while the colors on the background indicate interference.

We compared our result with the layout created using commercial software (see Fig. 4). Our layout allows for an extra $0.57 \%$ Annual Energy Production (AEP) which, in the lifetime of a wind park, equates to more than $6 \mathrm{M} €$ of extra income (net present value).

\subsection{Considering Cost of Foundations}

Costs of foundations can be a key factor, when the seabed conditions highly vary on the site. Due to waves, soil type and water depth, constructing a wind turbine in some positions of the site, could imply high extra costs. It is therefore valuable to include these costs in the optimization. To do so, we used the following strategy. Engineers from Vattenfall provided a cost map for the site: each possible position is associated with a construction cost,which was computed considering the foundation type, the weight of the turbine, the soil conditions for the specific position, and the water depth. Figure 5 shows the cost map for the site in hand (Borssele 1).

We slightly modified the objective function of model (1)-(8) as follows:

$$
\max \sum_{i \in V}\left(\left(P_{i}-\frac{c_{i}}{K_{\text {euro }}}\right) x_{i}-w_{i}\right)
$$

where $c_{i}$ is the price of constructing a turbine in position $i^{\prime}$ th (as specified on input) and $K_{\text {euro }}$ is a factor to scale the price from $€ / \mathrm{KW}$ to MW. To be specific, $K_{\text {euro }}$ is the cost for each MW of production considering a park lifetime of 25 years and a Weighted Average Cost of Capital (WACC) of $8 \%$. Both $c_{i}$ and $K_{\text {euro }}$ are problem specific and provided by Vattenfall.

Considering the same constraints as before and the same input data, but now including also foundation costs, we obtained the layout of Fig. 5.

The cost of foundations was previously not considered by any commercial software used by Vattenfall. Therefore, the layout was usually defined based only on AEP, and eventually some turbines located in too expensive positions were manually moved, obtaining a suboptimal layout. Figure 6 shows a comparison between our layout and the one provided by the company. Company experts verified that our layout allows for an extra $0.28 \%$ production, while decreasing the cost of foundations of more than $10 \mathrm{M} €$. All in all, they estimated an increased income of more than 12 $\mathrm{M} €$ over the wind farm lifetime.

Fig. 2 Borssele area, The Netherlands 
Fig. 3 Borssele Site I involving 50 Siemens $7 \mathrm{MW}-154$ turbines at a minimum distance of 5 rotor diameters. Colors in the background represent interference over all the possible wind scenarios in input, considering their frequency (color figure online)

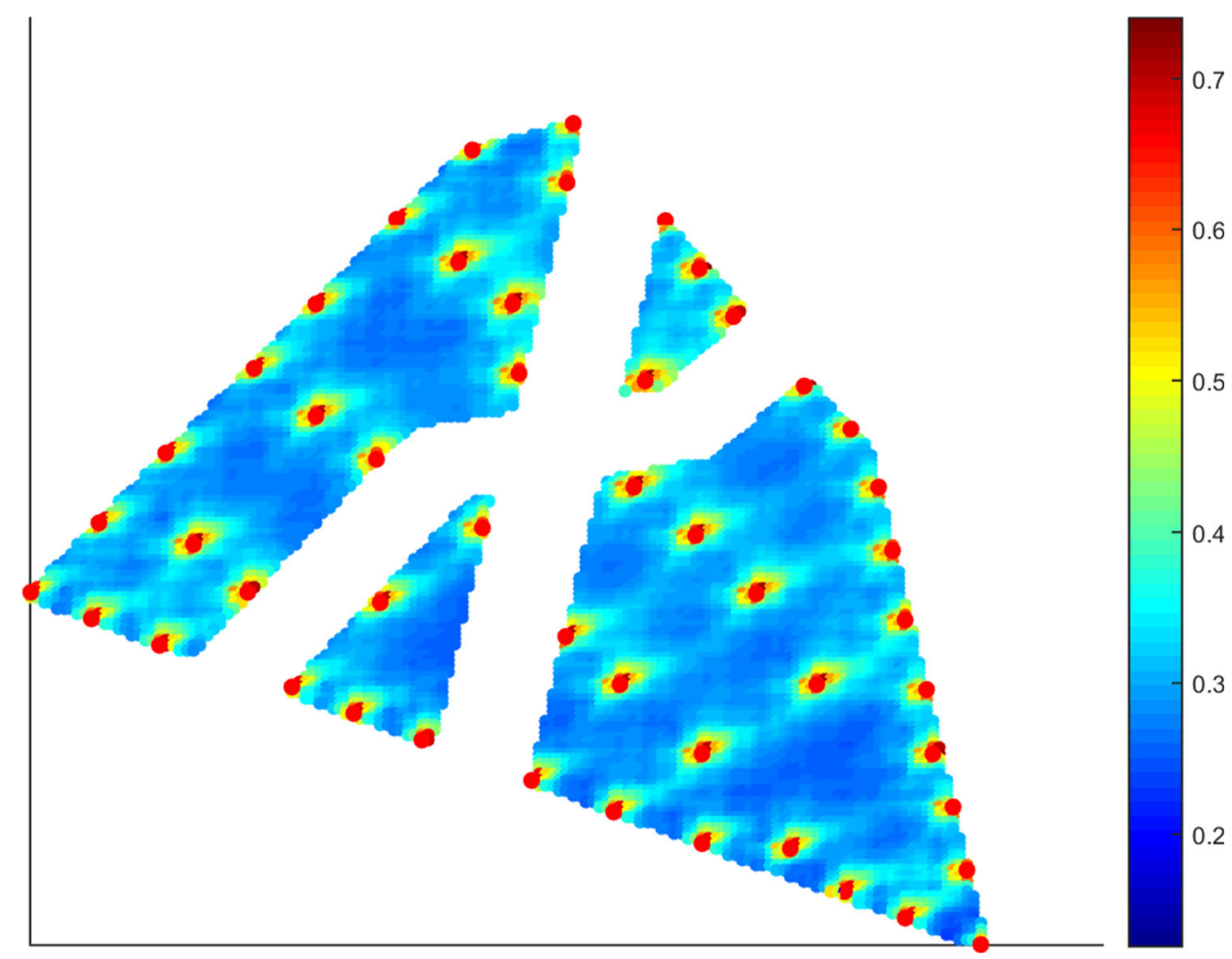

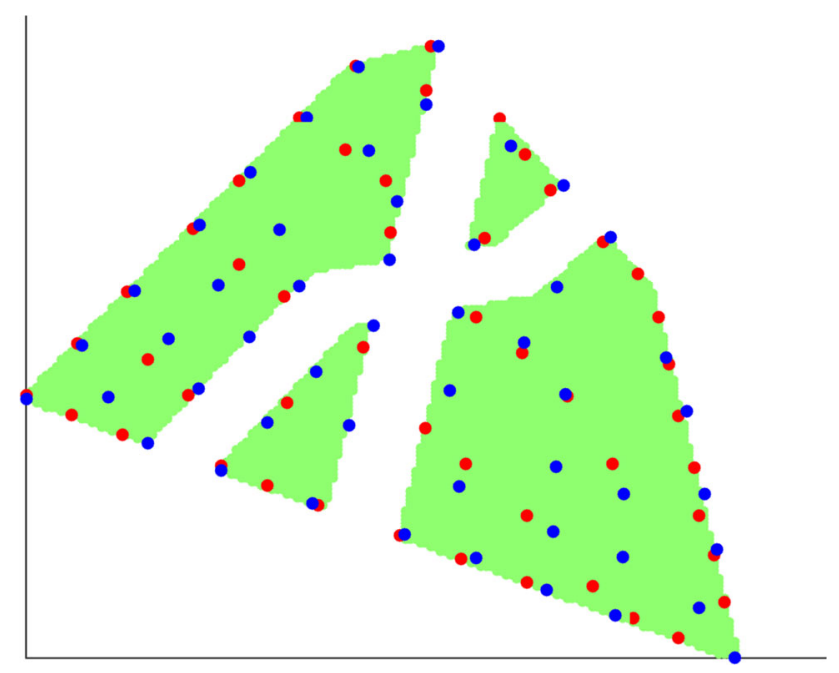

Fig. 4 Vattenfall layout (blue) vs Optimized layout (red) (color figure online)

The Borssele example clearly shows the potential of using mathematical optimization techniques as an integrated part of designing offshore wind parks.

\section{Matheuristics for Cable Routing}

We now assume that the turbine layout has been optimized and fixed, and we wish to find optimal cable connections between all turbines and the given collection point offshore [i.e., the substation(s)], minimizing the total cable costs. The optimization problem considers that:

- the energy leaving a turbine must be supported by a single cable;

- the maximum energy flow (when all the turbines produce their maximum) in each connection cannot exceed the capacity of the installed cable;

- different cables, with different capacities, costs and electrical resistances, can be installed;

- cable crossing must be avoided;

- a given maximum number of cables can be connected to each substation;

- cable losses (dependent on the cable type, the cable length and the current flow through the cable) must be considered.

Figure 7 illustrates a possible cable routing.

Following Fischetti and Pisinger (2017c), we model turbine positions as nodes of a complete and loop-free directed graph $G=(V, A)$, and all possible cable connections between them as directed arcs. Some nodes correspond to the substations that are considered as the roots of the trees, being the only nodes that collect energy. Let $P_{h}$ be the power production at node $h$. We distinguish between two different types of node: $V_{T}$ is the set of turbine nodes, and $V_{0}$ is the set of substation nodes. Let $T$ denote the set of different cable types that can be used. Each cable type $t \in T$ has a given capacity $k_{t}$ and unit cost $u_{t}$, representing the cost per meter of cable - immediate costs, i.e., capital 
Fig. 5 Foundation costs map for Borssele 1, and optimized solution considering AEP and foundation cost: different colors represent different costs to built a turbine in the specific position. Black dots represent turbines, while background colors show the foundation costs, where red colors are most expensive (the exact values are hidden for confidentiality reasons) (color figure online)

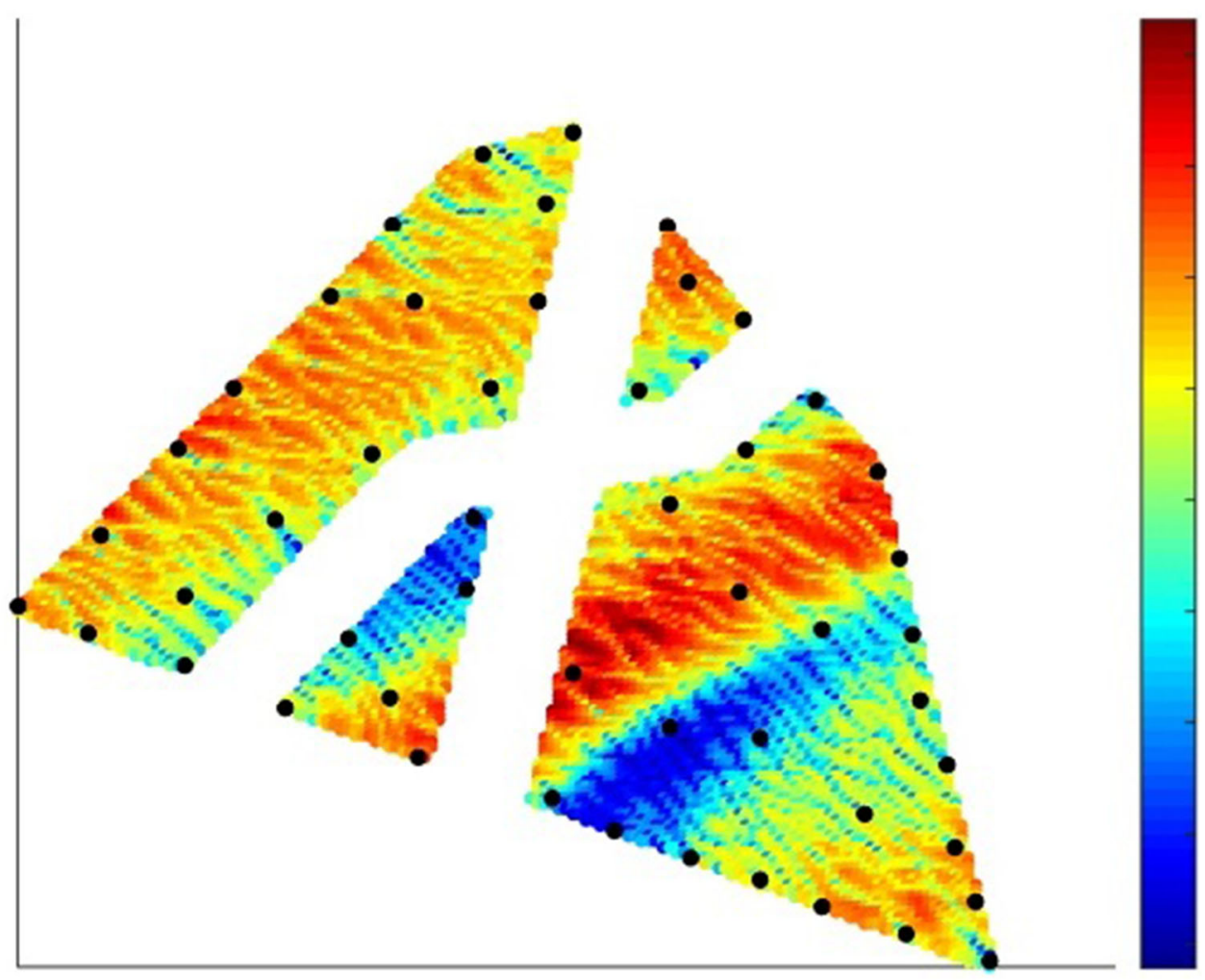

expenditure (CAPEX). Arc costs can therefore be defined as $c_{i, j}^{t}=u_{t} \operatorname{dist}(i, j)$ for each arc $(i, j) \in A$ and for each type $t \in T$, where $\operatorname{dist}(i, j)$ is the Euclidean distance between turbine $i$ and turbine $j$. The model uses the continuous variables $f_{i, j} \geq 0$ for the flow on arc $(i, j)$. Cable connections are defined by the binary variables $x_{i, j}^{t}$ which are 1 if and only if arc $(i, j)$ is connected with cable type $t$. Finally, variables $y_{i, j}$ indicate whether turbines $i$ and $j$ are connected (with any type of cable). Note that variables $y_{i, j}$ are related to variables $x_{i, j}^{t}$ as $\sum_{t \in T} x_{i, j}^{t}=y_{i, j}$. The overall model from Fischetti and Pisinger (2017c) is:

$\min \sum_{i, j \in V} \sum_{t \in T} c_{i, j}^{t} x_{i, j}^{t}$

s.t. $\sum_{t \in T} x_{i, j}^{t}=y_{i, j}, \quad i, j \in V, j \neq i$

$\sum_{i: i \neq h}\left(f_{h, i}-f_{i, h}\right)=P_{h}, \quad h \in V_{T}$

$\sum_{t \in T} k_{t} x_{i, j}^{t} \geq f_{i, j}, \quad i, j \in V, j \neq i$

$\sum_{j: j \neq h} y_{h, j}=1, \quad h \in V_{T}$

$\sum_{j: j \neq h} y_{h, j}=0, \quad h \in V_{0}$ $\sum_{i \neq h} y_{i, h} \leq C, \quad h \in V_{0}$

$x_{i, j}^{t} \in\{0,1\}, \quad i, j \in V, t \in T$

$y_{i, j} \in\{0,1\}, \quad i, j \in V$

$f_{i, j} \geq 0, \quad i, j \in V, j \neq i$.

The objective function (15) minimizes the total cable layout cost. Constraints (16) impose that only one type of cable can be selected for each built arc, and defines the $y_{i, j}$ variables. Constraints (17) are flow conservation constraints: the energy (flow) exiting each node $h$ is equal to the flow entering $h$, plus the power production of that node (except if the node is a substation). Constraints (18) ensure that the flow does not exceed the capacity of the installed cable, while constraints (19) and (20) impose that only one cable can exit a turbine and none can exit the substations (tree structure rooted at the substations). Finally, constraints (21) impose the maximum number of cables (C) that can enter each substation.

In order to model no-cross constraints we need a constraint for each pair of crossings arcs, i.e., a very large number of constraints. We have therefore decided to generate them on the fly, as also suggested in Bauer and Lysgaard (2015). In other words, the optimizer considers model (15)-(24) and adds the following new constraints whenever two established connections $(i, j)$ and $(h, k)$ cross 


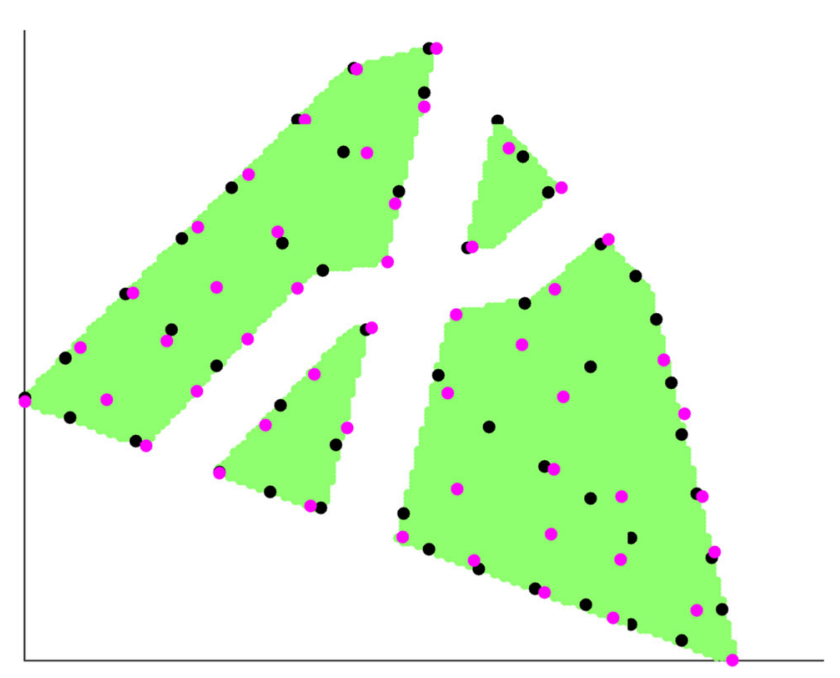

Fig. 6 Optimized layout considering wake effect and costs of foundations (black) versus Vattenfall layout (pink) (color figure online)

$y_{i, j}+y_{j, i}+y_{h, k}+y_{k, h} \leq 1$

The reader is referred to Fischetti and Pisinger (2017c) for stronger versions of those constraints. Using this approach, the number of non-crossing constraints actually added to the model decreases considerably, making the model faster to solve. Again, also in this application, the size of the problem is a main issue. As presented, the model is able to deal with small instances only, failing to find even a first feasible solution for large-size real-world instances. In order to produce high quality solutions in an acceptable amount of time also for large-scale instances, a matheuristic framework [as the one proposed in Fischetti and Pisinger (2017c)] can be used on top of this basic model. The main ideas behind our matheuristic framework are the following.

First, as we have already discussed previously, we know that warm starting the MILP solver with an initial solution can boost the resolution of large-size problems. In this application in particular, we decided to generate the first feasible solution using the MIP solver itself but on a relaxed version of the model. In the relaxed version of the model we allow for disconnected solutions, highly penalizing them in the objective function. Standard MILP solvers used on the relaxed model can quickly find a first (often disconnected) solution.

Secondly, we noticed that the difficulty of our problem was due to the large number of variables, i.e., the large number of possible cable connections in the complete directed graph. On the other hand, we also noticed that, once some arcs are fixed in the solution, the number of variables to optimize was highly reduced due to the nocross constraint. From these observations, we designed the following hybridization of exact mathematical modeling and heuristics (i.e., matheuristic): we define a first feasible solution $\left(x^{*}, y^{*}\right)$ using the MILP solver on the relaxed model, then we fix to 1 some of the $y$ variables with $y_{i, j}^{*}=1$. As said, fixing some arcs implies to exclude all the crossings arcs, with a drastic reduction in the dimension of the model. In order to decide which arcs to fix in the solution, we used different heuristic strategies, namely random fixing, string-based fixing, distance-to-substation fixing and fixing by sectors. For the sake of space we decided not to include more details on the heuristics here; the reader is referred to Fischetti and Pisinger (2017c) for more information. So, at each iteration, we temporarily fix to 1 some $y$ variables and apply the preprocessing described above to temporarily fix some other $y$ variables to zero. We then apply the MILP solver to the corresponding restricted problem, and we warm start the solver by providing the current solution $\left(x^{*}, y^{*}\right)$. We abort the execution as soon as a better solution is found, or a short time limit of a few seconds is reached. Then all fixed variables are unfixed, and the overall approach is repeated until a certain overall time limit (or maximum number of trials) is reached. Finally, the exact MILP solver is applied to the original model without any heuristic variable fixing, using the best-available solution to warm-start the solver.

\subsection{Real-World Application}

As a practical illustration we consider the cable routing of the existing wind park of Horns Rev 1, a real-world offshore park located in Denmark. Figure 8 (Kristoffersen and Christiansen 2003) shows the actual design for Horns Rev 1.

Three different types of cables can be used: the thinnest cable supports one turbine only, the medium supports 8 turbines, and the thickest 16. Based on the cable cross section, we estimated the costs and resistances of these cables. The estimated prices are 85,125 and $240 € / \mathrm{m}$, respectively, plus an estimated $260 € / \mathrm{m}$ for installation costs (independent of the cable type). Using the matheuristic techniques of Sect. 3 on this case, we obtained the layout in Fig. 9. The optimized layout is significantly different from the existing one: in terms of immediate costs, the optimized layout is more than 1.5 M€ less expensive.

\subsection{Cable Losses}

When the energy passes through a cable, there is a loss due to the electrical resistance of the cable. Different types of cables with different electrical resistances are available on the market. Therefore, one should aim at minimizing not only the immediate costs (CAPEX) but also the future 
Fig. 7 An example of cable routing: all turbines (black dots) are connected with one of the two substations (red squares). Please refer to the online paper for a color version of the figure

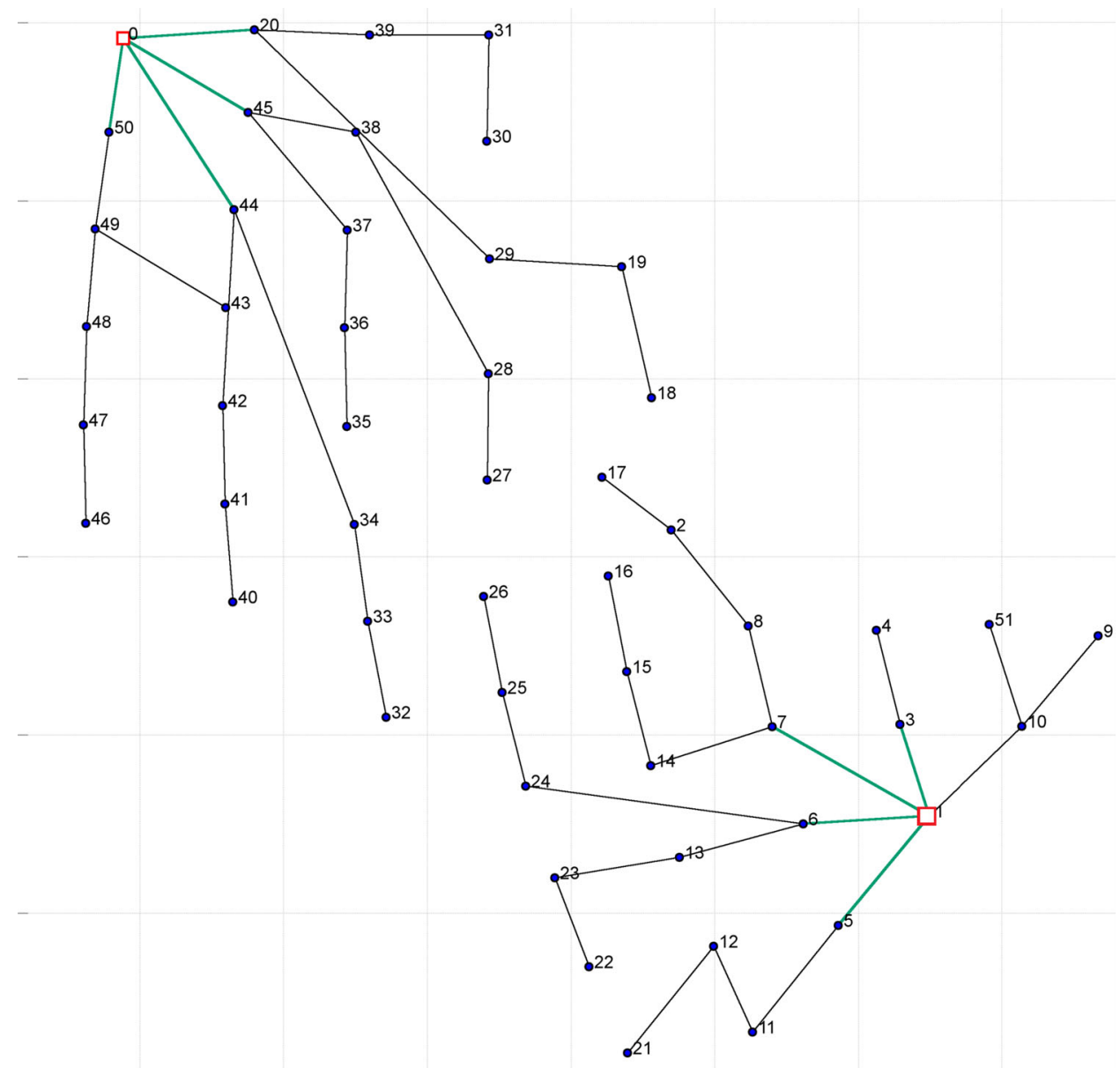

revenue losses due to power losses. This latter aspect is very important in practice, in that more expensive cables/ layouts can be significantly more profitable in the long run. This issue is explicitly considered in Fischetti and Pisinger (2017c), where a precomputing strategy is developed to include the losses in the optimization without increasing too much the size of the model. The main idea is that the current loss on a cable can be computed by knowing the (discrete) number of turbines connected to that specific cable. Due to the limited capacity of the cables, the revenue loss due to cable losses for each possible combination of cable type and number of turbines connected can be precomputed. As a result, by just changing the input prices of the cables, one can consider revenue losses without any change in the MILP model; see Fischetti and Pisinger (2017c) for details. In Fischetti and Pisinger (2017b) the authors analyze the impact of considering cable losses in real-world instances. The results show that, in some cases, several hundred thousand euros can be saved in the long run for a single cable routing, when considering losses already in the design phase (compared with a layout optimized on immediate costs only). Here, we still use the
Horns Rev 1 example we introduced previously, to give an illustration of the potential savings with respect to a manual (existing) layout.

As we have already seen, without considering losses in the optimization, the optimized layout for Horns Rev 1 would look as in Fig. 9. We can assume that the company decides to use this layout, making it possible to a-posteriori compute the losses related to it. It is still more profitable (by about 1.6 M€) than the existing one.

By optimizing cable losses, however, one can further improve its value in the long term. Figure 10 shows the optimized solution considering losses (thus optimizing the value of the cable route in its lifetime). Compared with the existing layout (Fig. 8), this new layout is about $1.7 \mathrm{M€}$ (NPV) more profitable in 20 years, and still around 1.5 M€ cheaper at construction time.

Table 1 summarizes the savings of the two optimized layouts compared with the existing one, both from an immediate cost perspective and from a long-term perspective.

The Horns Rev 1 example shows the impact of using mathematical optimization models for real world cable 
Fig. 8 Existing cable routing for Horns Rev 1 (Fischetti and Pisinger 2017b)

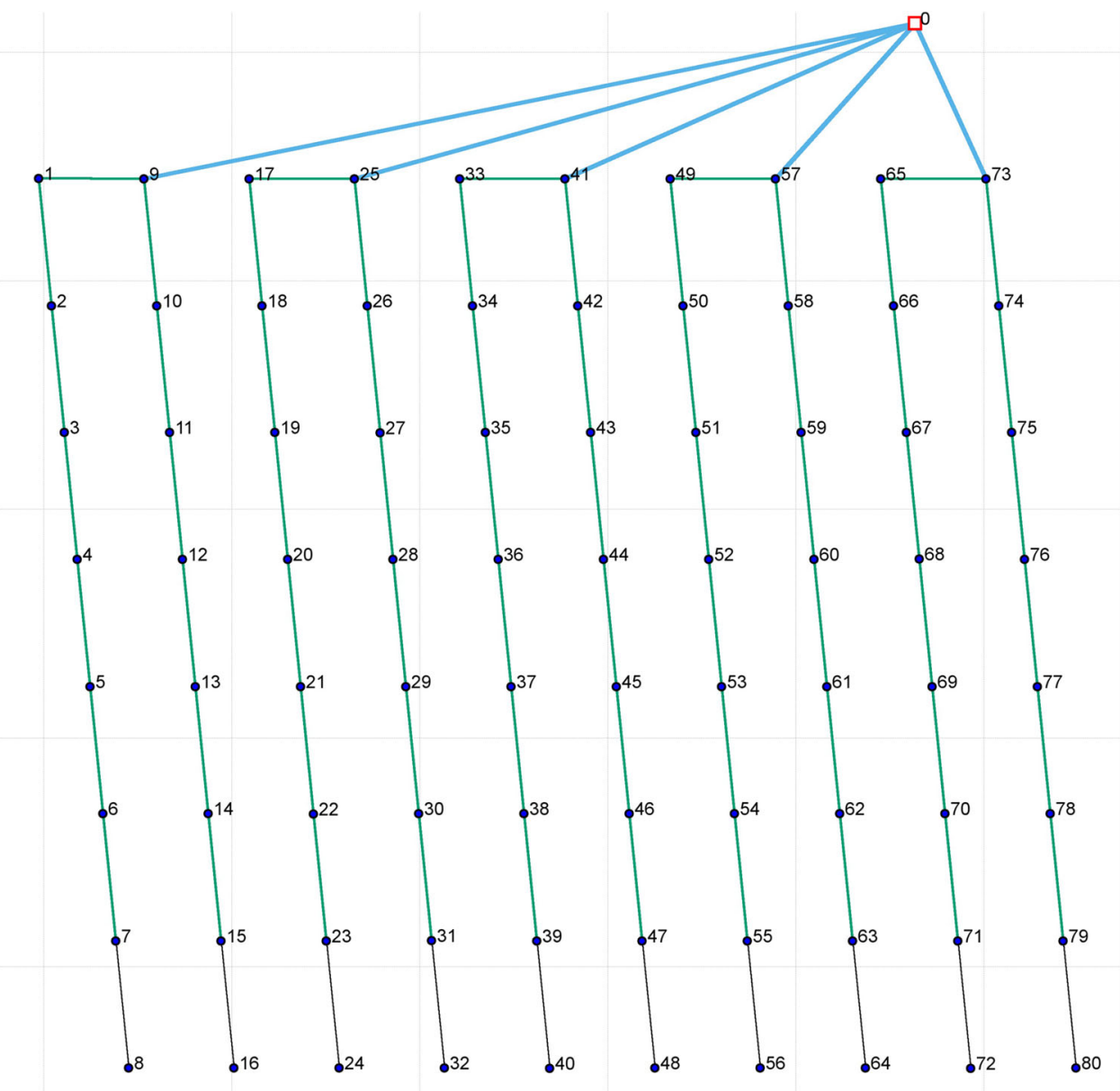

routing. The optimized layout is more than 1 M€ less expensive than the original (manual) one. Our model can be further extended to include additional constraints. Having a model where new constraints can easily be added is a key feature in a fast developing field of application as the wind-energy one. In Fischetti and Pisinger (2017a) it was shown that this model can be extended to consider e.g., a maximum number of branches, loop structures to reduce the risk of cable failures and use of new technologies on the market. They also show the potential savings in considering (or not considering) these additional constraints in practice. Our optimization tool is able to solve real-world instances in a matter of minutes, allowing for different what-if analyses. Being able to quantify the impact of a design choice and to conduct a fast what-if analysis are key features for Vattenfall, all of which being impossible without a proper optimization tool.

\section{Jacket Foundation Optimization}

As wind farms are getting larger and more remotely located, installation and infrastructure costs are rising. In particular, offshore turbines are getting bigger and bigger, and heavier foundations are required.

Different foundation types exist, depending on the seabed conditions and on the turbine size; see Fig. 11 for an illustration. In this section we focus on jacket (or space frame) foundations, which are one of the most complex/expensive structures, normally used at high water depth or for difficult soil conditions.

Once constructed, the foundation structures must resist stresses caused by the weight of the turbine, the wind that impacts it and the wave/currents in the sea area. More specifically, when designing a jacket structure, the designer has to choose a set of appropriate dimensions for the structural tubes for the space frame. The tube sizes, i.e., diameter and wall thickness, are chosen such that the joints can withstand the stresses that arise due to the loads. If the tube dimensions are chosen too slender, the stresses would exceed threshold values, leading to premature fatigue failure. On the other hand, if the tube dimensions are chosen too big, the tubes will be under-utilized and the overall structure will be too heavy and expensive. Thus, it is a delicate balance to find the optimal selection of tube dimensions. 


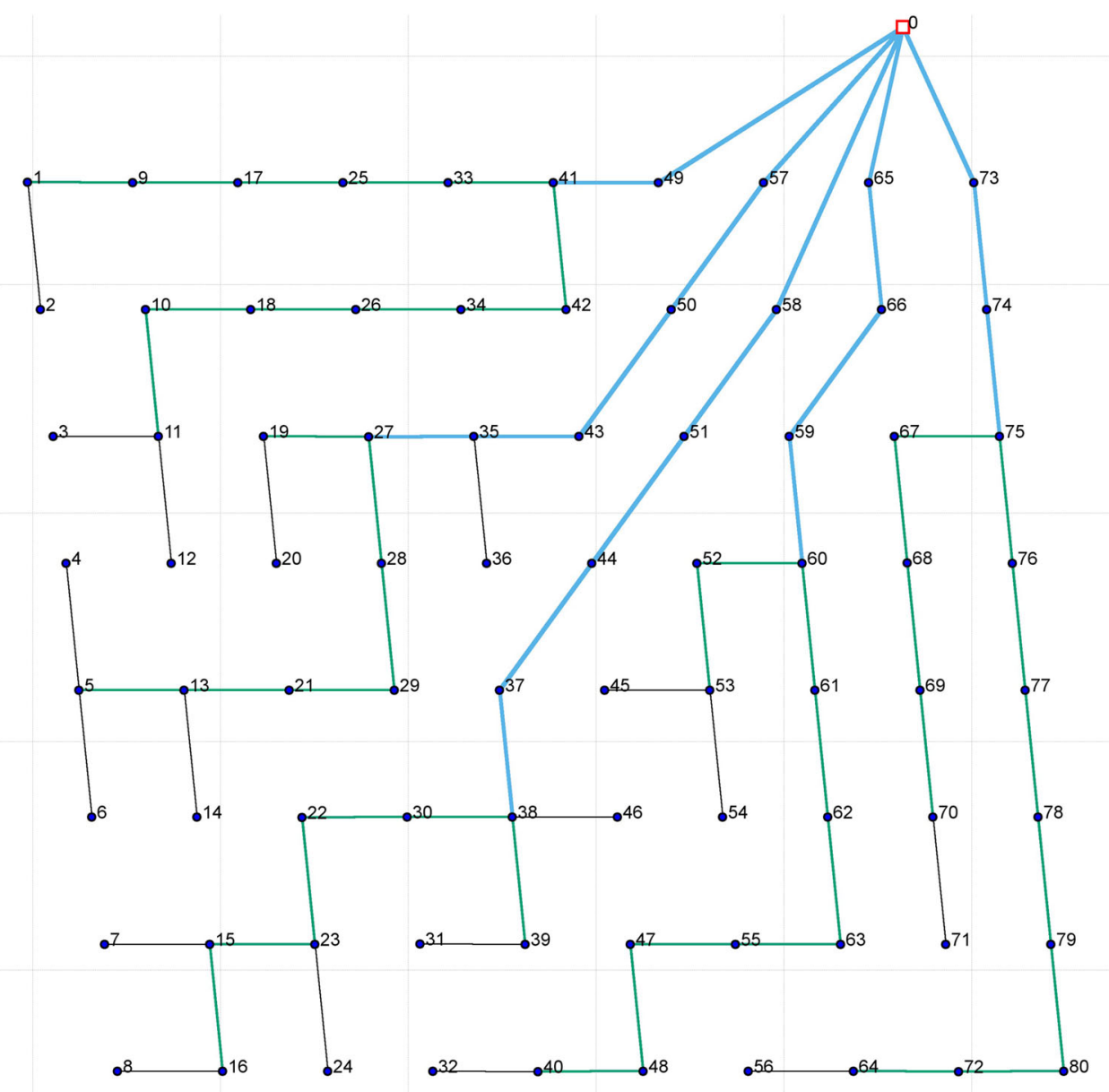

Fig. 9 Optimized layout for Horns Rev 1 (CAPEX costs only): this layout is more than 1.5 M€ cheaper than the existing one (Fischetti and Pisinger 2017b)

When optimizing it is therefore of crucial importance to consider these forces; for such a measure, we used the DNV-GL standards commonly used by practitioners (DNV-GL 2005).

A jacket foundation is identified by a structure, i.e., by a collection of joints and tubes. Tubes are called chords or braces. Chords are the main vertical columns carrying the overall loads and are normally of bigger dimensions. The horizontal and diagonal tubes are called braces and act as stiffeners. Their dimensions are usually smaller than the chords. A chord is connected with one or more braces by welding. Joints can be of different types, depending on how many tubes are connected through them. As all the faces of the jacket are identical, it is common practice to visualize the $3 \mathrm{D}$ structure as its $2 \mathrm{D}$ projection. Looking at a $2 \mathrm{D}$ representation of a structure, it is easy to identify the joint types: T-types connect a chord and a brace, K-types connect a chord and 2 braces, and X-types connect two braces. Figure 12 shows a 2D representation of a jacket foundation.
The company experts provided a list of possible tube types as input data: each of them has a specific diameter $(\mathrm{mm})$, a specific thickness $(\mathrm{mm})$ and unit mass $(\mathrm{kg} / \mathrm{m})$. These tube types come from a standard list offered by the tube manufacturer. In principle, the company could require the manufacturers to design customized tubes, but this has an extra cost; this is why, the aim is to use only standard tubes, leading to great savings. The optimization task consists in optimally selecting these standard components, minimizing the total structure cost, while ensuring no premature fatigue failures.

\subsection{The Optimization Model}

Input data includes the shape of the jacket foundation to be built (i.e., the joints, chords and braces and the way they are connected) and a set of tube types $T$. Each tube type $t \in T$ has a different mass, say $m_{t}$. We aim at optimizing the tube selection in order to minimize the total mass of the structure, subject to the following requirements: 
Fig. 10 Optimized layout for Horns Rev 1 (considering losses): in the 20-years wind park lifetime this layout is estimated to be more than 1.7 M€ more profitable than the existing one Fischetti and Pisinger (2017b)

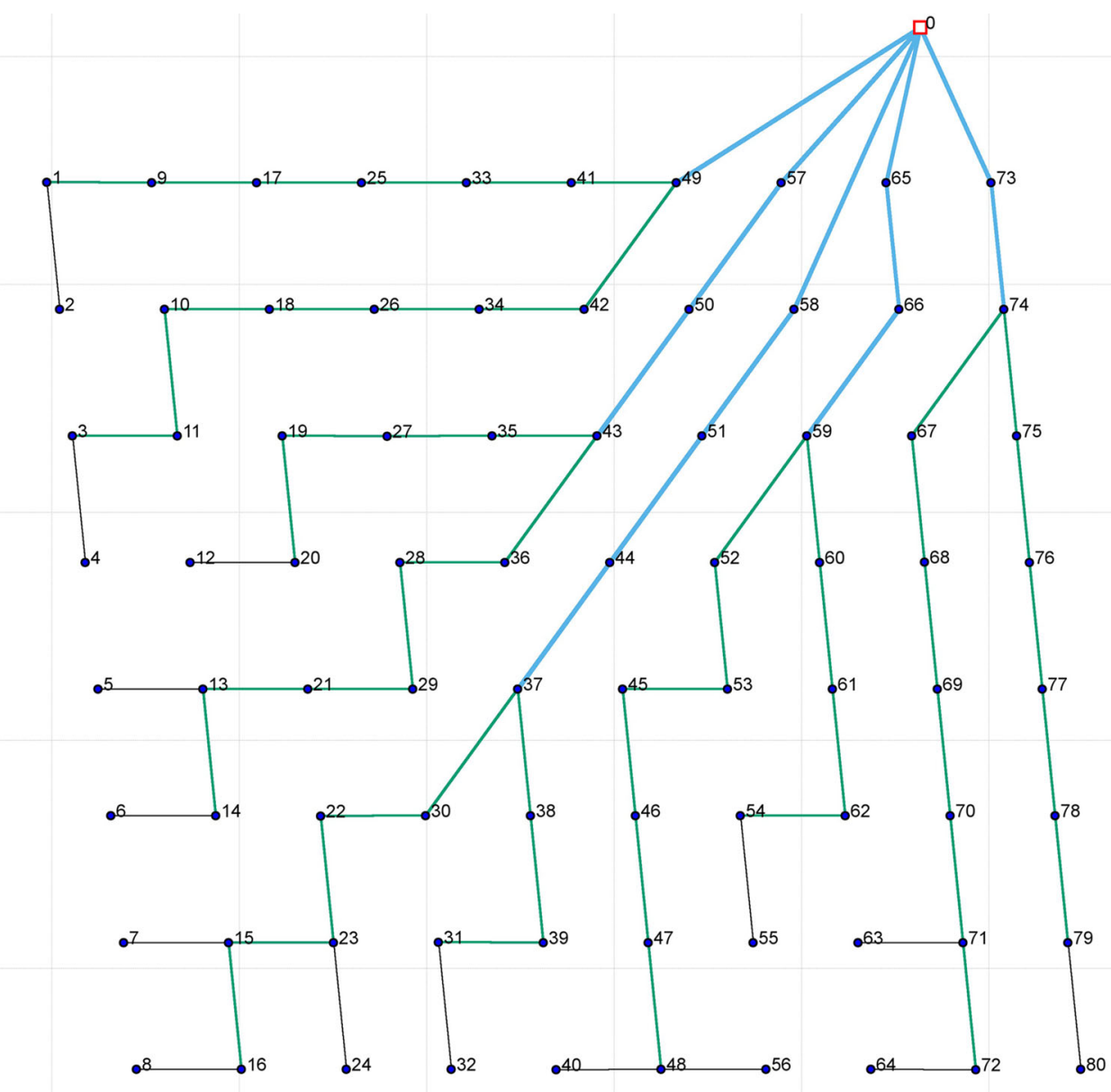

Table 1 Savings of optimized solutions compared with the existing cable routing for Horns Rev 1 (Fischetti and Pisinger 2017b)

\begin{tabular}{lll}
\hline Opt mode & Immediate & In 25 years \\
\hline Savings $(\mathrm{M} €)$ & & \\
CAPEX & 1.54 & 1.60 \\
Lifetime & 1.51 & 1.68 \\
\hline
\end{tabular}

- the tube type should be able to handle the local stresses (damage constraint);

- chord tubes should have a larger diameter than brace tubes;

- only one tube type should be selected for each connection.

The problem is naturally formulated on a directed graph $G=(V, A)$ where the set of nodes $V$ contains all the joints, and the set of $\operatorname{arcs} A$ contains all the tubes. We can then define a binary variable $x_{a}^{t}$ for each $a \in A$ and $t \in T$, where $x_{a}^{t}=1$ iff arc $a$ has a tube of type $t$. Different forces will act on each arc. In particular, if two generic nodes $i$ and $j$ are connected through an arc $a$, this arc will cause different loads on $i$ and $j$. To capture this in our model, we created a copy of all the given arcs, directing them so that we associated to $a=(i, j)$ the forces acting on $j$ because of the $a$ connection, while we associate to its symmetric arc $a^{\prime}=$ $(j, i)$ the forces acting on $i$ because of $a$. Note that arc orientation is only conventional, in that only one tube actually exists in the jacket structure (so we impose that $\left.x_{a}^{t}=x_{a^{\prime}}^{t}\right)$. Our MILP model then reads

$$
\begin{aligned}
& \min \frac{1}{2} \sum_{a \in A} \sum_{t \in T}\left(l_{a} m_{t}\right) x_{a}^{t} \\
& \text { s.t. } \sum_{t \in T} x_{a}^{t}=1 \quad a \in A \\
& x_{a}^{t}-x_{a_{1}}^{t}=0, \quad t \in T, \text { symmetric } \operatorname{arc} \text { pair }\left(\mathrm{a}, \mathrm{a}_{1}\right) \in \mathrm{A}^{2} \\
& x_{a}^{t}+x_{a_{1}}^{t_{1}} \leq 1, \quad\left\{a, a_{1}, t, t_{1}\right\} \in \bar{T}, \\
& \quad\left\{a, a_{1}\right\} \text { in a T-joint with } a \neq a_{1}, t, t_{1} \in T \\
& \quad x_{a}^{t}+x_{a_{1}}^{t_{1}}+x_{a_{2}}^{t_{2}} \leq 2, \quad\left\{a, a_{1}, a_{2}, t, t_{1}, t_{2}\right\} \in \bar{K},
\end{aligned}
$$


Fig. 11 Different types of foundation - image from EWEA (2012)
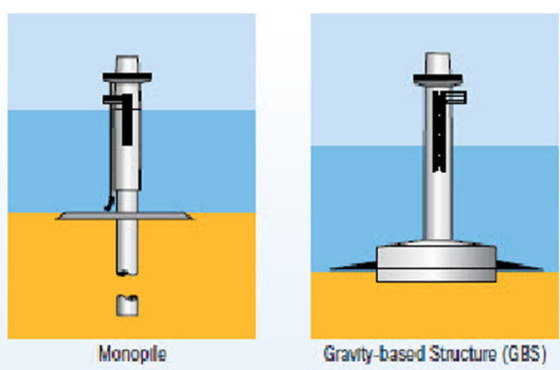

Manople

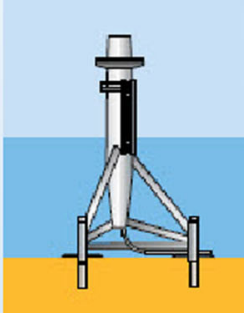

Space Frame (Tripod)

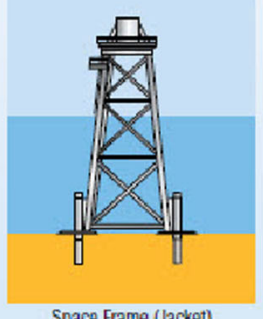

Space Frame (Jacket)

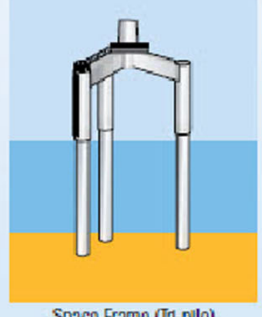

Space Frame (In-plle)

Sounce: EWEA

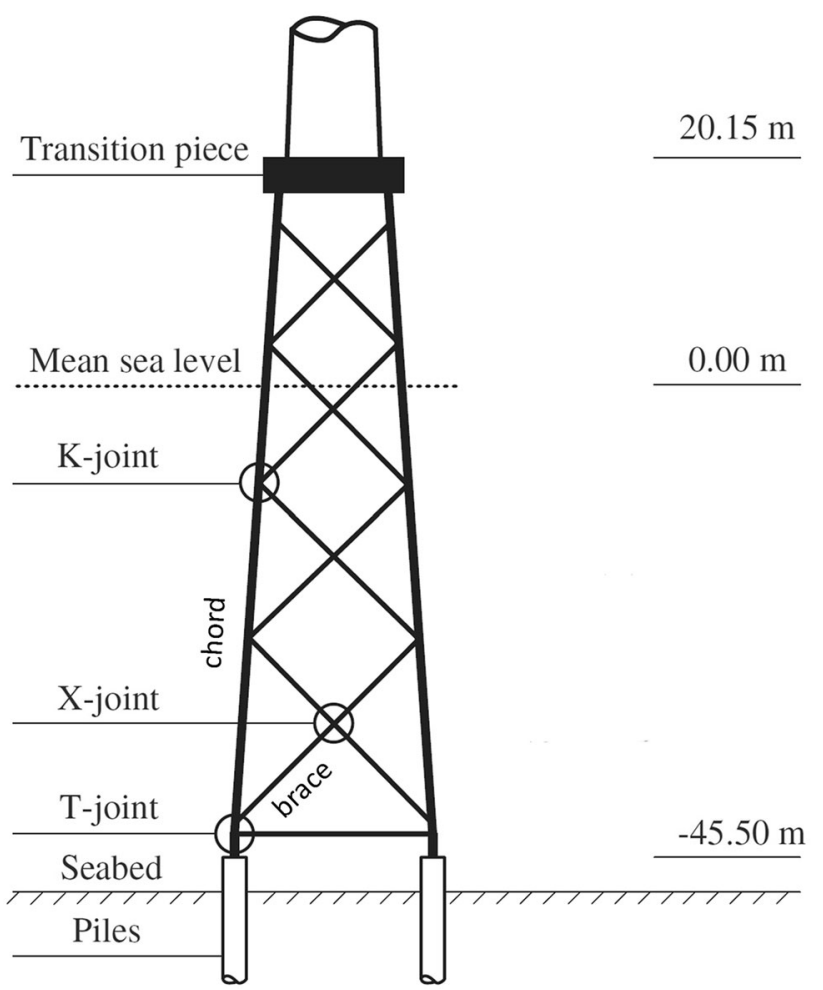

Fig. 12 Basic components of a jacket foundation

$\left\{a, a_{1}, a_{2}\right\}$ in a K-joint with $a \neq a_{1} \neq a_{2}$,

$t, t_{1}, t_{2} \in T$

$x_{a}^{t} \in\{0,1\}, \quad a \in A, t \in T$.

In the objective function (26) we minimize the total mass structure. Note that the contribution of each single arc $a$ to the total mass of the structure is given by the unit mass of

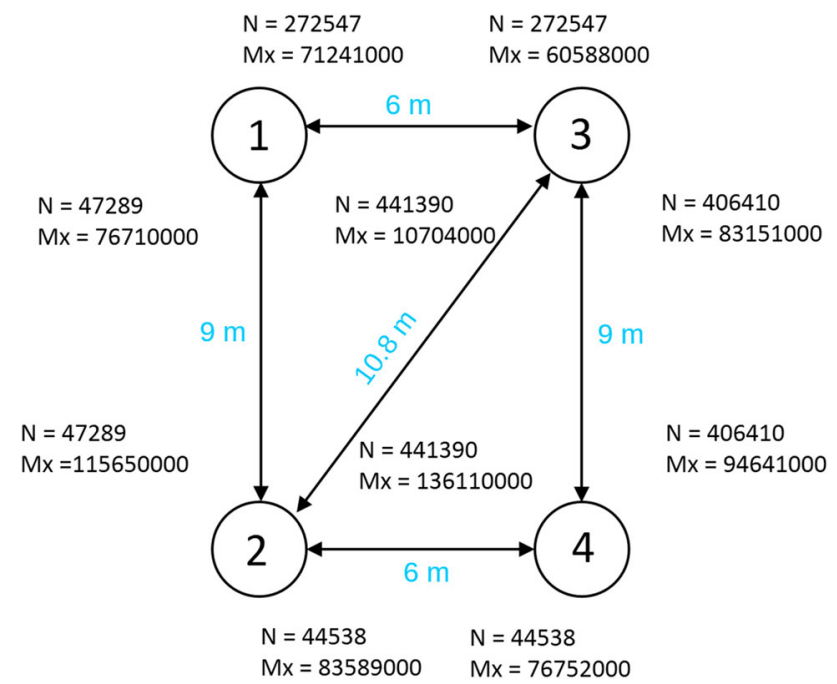

Fig. 13 Our illustrative example. The figure shows the structure of one face of the jacket foundation, the length of the tubes, and the different forces acting on them. $\mathrm{N}$ is the axial force in Newtons and the moment $\mathrm{Mx}$ is the inplane bending moment in Newtonmillimeters

the specific tube type $t$ selected (indicated as $m_{t}$ ) multiplied by the length of the arc $\left(l_{a}\right)$. Constraints (27) impose that one type of tube is selected for each arc in the structure, while constraints (28) impose that the same tube type is used for symmetric arcs. Constraints (29) and (30) forbid infeasible tube connections. In particular, set $\bar{T}$ contains all the pairs of arcs connected in T-connections that are infeasible due to limits on the damage levels. Analogously, set $\bar{K}$ in (30) refers to K-joints: it contains all the arcs connected in a $\mathrm{K}$-joint that are infeasible due to limits on 
Table 2 Possible tube types to use

\begin{tabular}{llc}
\hline Diameter $(\mathrm{mm})$ & Thickness $(\mathrm{mm})$ & Mass $(\mathrm{kg} / \mathrm{m})$ \\
\hline 400 & 30 & 278 \\
610 & 30 & 429 \\
610 & 40 & 562 \\
610 & 50 & 691 \\
711 & 30 & 504 \\
711 & 36 & 599 \\
711 & 45 & 739 \\
813 & 30 & 579 \\
813 & 40 & 763 \\
813 & 50 & 941 \\
1219 & 30 & 880 \\
1219 & 40 & 1163 \\
1219 & 50 & 1441 \\
1422 & 30 & 1030 \\
1422 & 40 & 1363 \\
1422 & 50 & 1692 \\
1626 & 30 & 1181 \\
1626 & 40 & 1565 \\
1626 & 50 & 1943 \\
\hline & &
\end{tabular}

Table 3 Solution of the tube selection optimization problem: the manual solution (left) vs the optimized one (right)

\begin{tabular}{llllll}
\hline Connection & Manual & & & \multicolumn{2}{l}{ Optimized } \\
\cline { 5 - 6 } \cline { 5 - 6 } & Diameter & Thickness & & Diameter & Thickness \\
\hline$(1,2)$ & 1219 & 50 & 1219 & 30 \\
$(3,4)$ & 1219 & 50 & 1219 & 30 \\
$(1,3)$ & 711 & 30 & 1219 & 30 \\
$(2,3)$ & 813 & 40 & & 1219 & 30 \\
$(2,4)$ & 610 & 30 & 813 & 30 \\
Total mass & 39.8 Tons & & 34.1 Tons \\
\hline
\end{tabular}

the damage levels. Both sets $\bar{T}$ and $\bar{K}$ include also pairs or triplets (respectively) where the braces are bigger than the chord (as required by Vattenfall's engineers). Finally (31) requires that all variables are binary. It can be noticed that the MILP model does not depend explicitly on the actual damage formulas, which are only used for the definition of the no good sets $\bar{T}$ and $\bar{K}$ [in our implementation, we used official standards in the field (DNV-GL 2005), but different formulas can be implemented as well].

\subsection{Preliminary Results}

For simplicity, we are applying the MILP model on a simple, but representative structure consisting of $\mathrm{T}$ and $\mathrm{K}$ joints. The structure is a simple structure, but the applied methodology is easily expandable to real life structures with more complex joints. As already mentioned, in a jacket foundation all the faces of the structure are identical, therefore the study is carried out on one of the faces, and then extended to the others. Therefore, we will next consider a $2 \mathrm{D}$ representation of our 3D jacket foundation example.

As already discussed in Sect. 4.1, a jacket foundation can be represented as a graph with joints as nodes and tubes as arcs. We will use this representation for our test example. We are given from Vattenfall's experts the structure of the foundation (i.e., the set of nodes and arcs) and we have to determine the tube type to be used for each arc. We are also given the different forces acting on each arc of the structure. Forces can be different from one side of the tube to the other, therefore we are given forces for each extreme of any physical connection (as shown in Fig. 13). Based on the sectional forces $\mathrm{N}$ and $M_{x}$ in Fig. 13, an expected fatigue lifetime of the joint is calculated based on a set of parametric joint formulas (DNV-GL 2005).

We are also given a set of possible tube types (as in Table 2) to use in each of the physical connections. Each tube type is characterized by its diameter, thickness and unit mass. The diameter and thickness of each tube impact its capacity of withstanding different forces. We aim at minimizing the total mass of the structure while ensuring that the structure can withstand the different forces acting on it.

We performed the tube type selection using our optimization model of Sect. 4.1, while an expert from Vattenfall performed the same task manually. The optimization solver (IBM ILOG Cplex 12.6) reached optimality in a matter of seconds. Table 3 shows a comparison with the manual solution. In the first column of Table 3 we report the arc, then we specify the tube selected manually (second and third column) or by the optimization model (fourth and fifth column). Finally, in the last table row, we compare the total mass of the two feasible solutions (both satisfying the damage constraints).

As it can be seen from the last row of Table 3, the optimized structure is much lighter than the manually constructed one (about 5 Tons less, with a saving of more than $12 \%$ ). This is a very interesting result: although the toy structure was really simple, the optimization could still significantly outperform the manual approach. We therefore expect to have even larger savings for more complex (real-world) structures, where the manual task is much more difficult to carry out. Furthermore, by having an automated process, the designers would be more willing to perform additional design iterations, since they do not have to carry out the tedious optimization job manually for each design iteration. 


\section{Conclusions}

In order to make wind energy competitive with non-renewable energy sources, every part of an offshore wind farm must be optimized to improve efficiency and reduce costs. In this overview we have shown how mathematical optimization can significantly improve several steps of the design phase. In particular we have addressed turbine allocation, inter-array cable routing and optimization of jacket foundations. Given the large size and complexity of the instances, matheuristic techniques have been developed and used to optimize real-world wind farms. We have shown that millions of Euros can be saved in this way. Using MILP-based models, rather than manual solutions, we have also been able to quantify the impact of different design choices and to carry out different what-if analysis (for example considering power losses in cable routing, or considering cost of foundations in the wind park layout). This is extremely interesting from an application perspective, in that it allows the company to have a better understanding of the case in hand and to take informed decisions.

Still, many optimization challenges have not been solved in the wind field. Looking at the problems we considered in this overview, an interesting next step would be to look at the integration of the different optimization phases in wind park design. As we have seen, the wind farm layout model tends to spread turbines as much as possible, in order to reduce wake effect. On the other hand, the further apart the turbines are located, the higher becomes the infrastructure costs to connect them. It would therefore be interesting to integrate, for example, the optimization of wind farm layout and cable routing together. Given the large size and complexity of both problems, some challenges would arise in solving a unified mode.

The models presented in this overview could also be generalized to onshore parks. Onshore wind park design is more complex, as it includes some additional constraints and non linearities. In particular, most of the work on wind farm layout optimization assumes the wind to blow uniformly in the site. This assumption does not hold in onshore sites, where the shape of the land (mountains, hills, forests, etc.) impacts the free-wind speed. Furthermore, some extra constraints must be taken into account in the onshore case, such as noise limitations for nearby houses, or road connections to the turbines.

Looking further ahead, the wind energy sector is quickly evolving, so new technologies have to be considered. An example could be the Offshore Transformer Modules (OTM) that just recently entered the market. Those transformers are meant to substitute offshore substations, and can be connected to the turbine directly. Each turbine equipped with an OTM, can be connected both to interarray cables and to higher-voltage cables (i.e., export cables). Considering OTMs in the cable routing optimization opens up for some new and interesting optimization tasks, such as deciding the number of OTMs in a park, deciding their position, etc. The presented MILP solution framework can easily incorporate new constraints, therefore it is very suitable for such a fast evolving field. Finally, floating wind farms are slowly appearing. Optimizing the establishment and operation of such wind farms will introduce many new challenges. In particular if the wind turbines can be moved slightly to reduce wake effect.

Acknowledgements The work of the first author was carried out as an industrial $\mathrm{PhD}$ project, supported by Innovation Fund Denmark and Vattenfall BA Wind, in collaboration with DTU Management. We would like to thank our industrial partner, and in the specific Jesper Runge Kristoffersen, Iulian Vranceanu, Thomas Hjort and Kenneth Skaug who helped us in defining the cable routing and wind park design constraints; and Per Christian Hyldahl who helped defining jacket foundation problem and testing our model.

\section{References}

Archer R, Nates G, Donovan S, Waterer H (2011) Wind turbine interference in a wind farm layout optimization-mixed integer linear programming model. Wind Eng 35(2):165-178

Barthelmie RJ, Hansen K, Frandsen ST, Rathmann O, Schepers JG, Schlez W, Phillips J, Rados K, Zervos A, Politis ES, Chaviaropoulos PK (2009) Modelling and measuring flow and wind turbine wakes in large wind farms offshore. Wind Energy $12: 431-444$

Bauer J, Lysgaard J (2015) The offshore wind farm array cable layout problem: a planar open vehicle routing problem. J Oper Res Soc 66(3):360-368

Berzan C, Veeramachaneni K, McDermott J, Reilly UMO (2011) Algorithms for cable network design on large-scale wind farms. Tufts University, Technical report

Boschetti MA, Maniezzo V, Roffilli M, Röhler Bolufé A (2009) Matheuristics: optimization, simulation and control. Hybrid metaheuristics. Springer, Heidelberg, pp 171-177

Dai L, Stlhane M, Utne IB (2015) Routing and scheduling of maintenance fleet for offshore wind farms. Wind Eng 39(1):15-30

DNV-GL 2005. Fatigue design of offshore steel structures. Recommended practice DNVGL-RP-C203:2014-06

Dutta S, Overbye TJ (2011) A clustering based wind farm collector system cable layout design. In: Power and energy conference at Illinois, pp 1-6

EWEA (2012) European Wind Energy Association. http://www.ewea. org/. Accessed 15 Jan 2012

Fagerfjall P (2010) Optimizing wind farm layout-more bang for the buck using mixed integer linear programming. Master's thesis, Department of Mathematical Sciences, Chalmers University of Technology and Gothenburg University, Goteborg

Fischetti M (2014) Mixed-integer models and algorithms for wind farm layout optimization. Master's thesis, University of Padova. http://tesi.cab.unipd.it/45458/1/tesi_Fischetti.pdf

Fischetti M, Monaci M (2014) Proximity search for 0-1 mixedinteger convex programming. J Heuristics 20(6):709-731

Fischetti M, Monaci M (2016) Proximity search heuristics for wind farm optimal layout. J Heuristics 22(4):459-474

Fischetti M, Pisinger D (2017a) Mixed integer linear programming for new trends in wind farm cable routing. In: ENDM special issue for INOC2017 (to appear) 
Fischetti M, Pisinger D (2017b) On the impact of using mixed integer programming techniques on real-world offshore wind parks. In: Proceedings of the 6th international conference on operations research and enterprise systems, Porto, pp 108-118

Fischetti M, Pisinger D (2017c) Optimizing wind farm cable routing considering power losses. Europ J Oper Res. (to appear)

Gundegjerde CI, Halvorsen B, Halvorsen-Weare EE, Hvattum LM, Nons LM (2015) A stochastic fleet size and mix model for maintenance operations at offshore wind farms. Transp Res Part C Emerg Technol 52(Supplement C):74-92

Gutierrez-Alcoba A, Ortega G, Hendrix EMT, Elin E (2017) A model for optimal fleet composition of vessels for offshore wind farm maintenance. In: Procedia Comput Sci, 108(Supplement C):1512 - 1521. International Conference on Computational Science, ICCS 2017, 12-14 June 2017, Zurich, Switzerland

Hou P, Enevoldsen P, Eichman J, Hu W, Jacobson MZ, Chen Z (2017) Optimizing investments in coupled offshore wind-electrolytic hydrogen storage systems in Denmark. J Power Sour 359:186-197

Hou P, Hu W, Soltani M, Zhang B, Chen Z (2016) Optimization of decommission strategy for offshore wind farms. In: 2016 IEEE power and energy society general meeting (PESGM), pp 1-5. IEEE

Irawan CA, Ouelhadj D, Jones D, Stlhane M, Sperstad IB (2017) Optimisation of maintenance routing and scheduling for offshore wind farms. Europ J Oper Res 256(1):76-89

Jensen, N.O. 1983. A note on wind generator interaction. Technical report, Technical Report Riso-M-2411(EN), Riso National Laboratory, Roskilde
Kristoffersen JR, Christiansen P (2003) Horns Rev offshore windfarm: its main controller and remote control system. Wind Eng 27(5):351-359

Kusiak A, Song Z (2010) Design of wind farm layout for maximum wind energy capture. Renew Energy 35(3):685-694

Kwong WY, Zhang PY, Romero D, Moran J, Michael M, Amon C (2012) Multi-objective optimization of wind farm layouts under energy generation and noise propagation. In: Proceedings of the ASME 2012 IDETC/CIE 2012, Chicago, pp 323-332

Muskulus M, Schafhirt S (2014) Design optimization of wind turbine support structures-a review. J Ocean Wind Energy 1(1):12-22

Oest J, Sorensen R, Overgaard LC, Lund E (2017) Structural optimization with fatigue and ultimate limit constraints of jacket structures for large offshore wind turbines. Struct Multidiscip Optimiz 55(3):779-793

Sinha S, Chandel SS (2015) Review of recent trends in optimization techniques for solar photovoltaicwind based hybrid energy systems. Renew Sustain Energy Rev 50(Supplement C):755-769

Topham E, McMillan D (2017) Sustainable decommissioning of an offshore wind farm. Renew Energy 102:470-480

Turner SDO, Romero DA, Zhang PY, Amon CH, Chan TCY (2014) A new mathematical programming approach to optimize wind farm layouts. Renew Energy 63(C):674-680

Zhang Peter Y, Romero David A, Christopher Beck J, Amon Cristina $\mathrm{H}$ (2014) Solving wind farm layout optimization with mixed integer programs and constraint programs. EURO J Comput Optimiz 2(3):195-219 